\title{
Buttiauxella noackiae
}

National Cancer Institute

\section{Source}

National Cancer Institute. Buttiauxella noackiae. NCI Thesaurus. Code C122255.

A species of facultatively anaerobic, Gram-negative, rod-shaped bacteria in the family Enterobacteriaceae. B. noackiae is positive for $\mathrm{N}$-acetyl-L-glutamine, L-arg inine dihydrolase, melibiose, and L-ornithine decarboxylase. 